\title{
Satisfação de pacientes quanto à assistência de enfermagem: dimensão educacional
}

\section{Patient's satisfaction as a nursing care: educational dimension}

Ághata Barbosa Ramalho Caldeira ${ }^{1}$, Valéria da Silva Baracho ${ }^{1,}$ Camila de Fátima Guedes ${ }^{1}$, Paulo Henrique da Cruz Ferreira ${ }^{2}$ Helisamara Mota Guedes ${ }^{3}$, Liliane da Consolação Campos Ribeiro ${ }^{3}$

1. Graduanda em Enfermagem pela Universidade Federal dos Vales do Jequitinhonha e Mucuri (UFVJM), Diamantina, Minas Gerais, Brasil. 2. Santa Casa de Caridade de Diamantina, Diamantina, Minas Gerais, Brasil. 3. Professora Adjunta do Departamento de Enfermagem da Universidade Federal dos Vales do Jequitinhonha e Mucuri (UFVJM), Diamantina, Minas Gerais, Brasil.

\section{Resumo}

Introdução: Conhecer a satisfação do paciente com os cuidados de enfermagem possibilita ao enfermeiro avaliar e planejar a assistência no sentido de atender às necessidades da clientela atendida. Objetivo: Este trabalho objetivou analisar a satisfação do paciente quanto à assistência de enfermagem hospitalar por meio da dimensão educacional. Método: Trata-se de um estudo descritivo realizado em um hospital privado, filantrópico, com 223 pacientes internados, utilizando o Instrumento de Satisfação do Paciente (ISP). Resultados: Encontrou-se a média de 3,62 no ISP. Para 70,9\% dos pacientes, o enfermeiro preocupa-se em explicar sobre a doença; $77,6 \%$ discordam de que o enfermeiro faça muitas perguntas sem utilidade; $85,7 \%$ relatam que esses profissionais não utilizam uma linguagem simples, uma vez que 83,9\% não entendem o que é falado por eles; $83,3 \%$ acham que os enfermeiros falam muito rápido; $68,6 \%$ gostariam que o enfermeiro desse explicações completas e suficientes acerca da solicitação de exames, e $48 \%$, dos resultados desses. Conclusão: Há necessidade de implementar ações de educação em saúde na instituição hospitalar, melhorar a comunicação entre pacientes e enfermeiros, para, assim, obter resultados benéficos que ajudem no tratamento do paciente e na qualidade assistencial.

Palavras-chave: Satisfação do Paciente. Qualidade da Assistência à Saúde. Educação em Enfermagem. Educação em Saúde. Cuidados de Enfermagem.

\begin{abstract}
Introduction: Knowing the patient's satisfaction with nursing care allows nurses to assess and organize assistance in order to meet the needs of the patients. Objective: This study aimed to analyze the patient's satisfaction in the hospital nursing care through educational dimension. Method: This is a descriptive study in a philanthropic hospital. The sample was composed of 223 hospitalized patients using the Patient Satisfaction Instrument (PSI). Results: We found an average of 3.62 at the PSI. To $70.9 \%$ of patients, the nurse is concerned to explain the disease, $77.6 \%$ disagree that the nurse asks many questions useless, $85.7 \%$ reported that professionals do not use simple language, and $83.9 \%$ do not understand that is spoken by nurses, $83.3 \%$ feel that nurses speak very fast and $68.6 \%$ would like the nurse to give a complete and sufficient explanation of the examination request and $48 \%$ of these results. Conclusion: There is the necessity of implementing the activities of health education to improve the communication between patients and nurses to get better outcomes in the patient's care.
\end{abstract}

Keywords: Patient's satisfaction. Quality of health care. Nursing education. Health education. Nursing Care.

\section{INTRODUÇÃO}

Com a globalização, todos têm fácil acesso às informações e tecnologias que estimulam o mercado a tornar-se cada vez mais competitivo, acirrado e atento à qualidade do serviço e à satisfação de seus clientes. Os hospitais estãose transformando em empresas que se preocupam com a qualidade do seu atendimento, sendo a satisfação do paciente um elemento principal para o feedback dessa qualidade ${ }^{1,2}$.

A satisfação do paciente reflete sua opinião sobre o atendimento recebido e se suas expectativas foram atendidas durante 0 período de internação, sendo este um importante indicador para o setor hospitalar a respeito da qualidade da assistência de enfermagem ${ }^{3}$.

A equipe de enfermagem é a principal responsável por sugestionar positiva ou negativamente a satisfação do paciente uma vez que é quem cuida, está ao lado do paciente durante toda a internação 24 horas por dia, além de representar a maior parte dos profissionais ${ }^{4}$.

As atividades desses profissionais no contexto da instituição hospitalar têm-se diversificado, tornando-se um processo complexo a ser desenvolvido em relação ao paciente e às suas necessidades, pois eles são compreendidos pelo cuidar, educar e gerenciar. Entretanto, na prática hospitalar, alguns profissionais possuem uma visão fragmentada e limitada, centrada apenas nos aspectos biológicos e tecnológicos, encontrando pouco tempo para o desenvolvimento de atividades de educação em saúde ${ }^{5}$.

É importante destacar que as ações educativas são imprescindíveis para a promoção à saúde e devem estar inseridas em todos os níveis de atenção à saúde do paciente; entretanto existem poucas publicações que enfocam a educação

Correspondência: Helisamara Mota Guedes. Universidade Federal dos Vales do Jequitinhonha e Mucuri - Campus JK, Departamento de Enfermagem. Rodovia MGT 367, Km 583, n 5000, Alto da Jacuba, CEP: 39100000 - Diamantina, MG - Brasil. E-mail: helisamaraguedes@gmail.com

Conflito de interesse: Não há conflito de interesse por parte de qualquer um dos autores.

Recebido em: 6 Jun 2016; Revisado em: 27 Jul 2016;Aceito em: 5 Ago 2016 
em saúde realizada por enfermeiros no contexto de internação hospitalar. Acredita-se que esta seja uma tendência emergente, que precisa ser discutida e estudada, a fim de promover a melhoria da qualidade assistencial ${ }^{6}$.

Neste sentido, este trabalho teve como objetivo analisar a satisfação do paciente quanto à assistência de enfermagem hospitalar por meio da dimensão educacional.

\section{MÉTODO}

Trata-se de um estudo descritivo, realizado em um hospital polo referência para a região ampliada de saúde, situado no alto do Vale do Jequitinhonha, em Minas Gerais. As entrevistas foram realizadas à beira do leito dos pacientes por meio da aplicação do Instrumento de Satisfação do Paciente (ISP), adaptado e validado à cultura brasileira ${ }^{7}$. A coleta de dados compreendeu o período entre agosto a outubro de 2014.

A população deste estudo constituiu-se de pacientes que estavam internados nas clínicas médica, cirúrgica, neurológica e convênios.

O cálculo amostral foi realizado no programa Diman 1.0, considerando $80 \%$ de taxa de ocupação mensal, com margem de erro de $5 \%$ e média de 358 clientes externos nos últimos três meses, acrescido de $10 \%$ de possíveis perdas. A amostra final foi composta por 223 entrevistados.

Para estratificar a amostra, foi obtida a relação do total de leitos por clínicas de internação a fim de distribuir, de modo proporcional, a real distribuição dos adultos por clínicas, sendo nove leitos nos convênios, 33 na médica, 15 na cirúrgica e 19 na neurológica.

A partir desse enfoque, foram utilizados os seguintes critérios de inclusão: devem estar de alta hospitalar com mais de 48 horas de internação, orientados em tempo e espaço; comunicando verbalmente; terem idade igual ou superior a 18 anos, sendo entrevistados os primeiros pacientes que apresentaram os critérios de inclusão de cada clínica, por mês.

O ISP possui 25 questões organizadas em três domínios: educacional, confiança e profissional, em formato de escalas tipo Likert com cinco opções: concordo totalmente, concordo, não tenho opinião, discordo e discordo totalmente ${ }^{8}$. Os resultados do instrumento variam em uma escala de um a cinco, sendo atribuída às conotações positivas a pontuação um para as respostas discordo totalmente e a pontuação máxima de cinco para as respostas concordo totalmente. Quanto maior a pontuação atingida, maior será o grau de satisfação do paciente ${ }^{7}$. As perguntas com conotação de negação têm sua pontuação invertida durante avaliação da escala, ou seja, pontuação um para as respostas concordo totalmente e pontuação cinco para as respostas discordo totalmente ${ }^{9}$.

Para cada item do instrumento, foi calculada a média das respostas, sendo considerado neste estudo como satisfeito a obtenção de um escore maior ou igual a três $(\geq 3)^{9}$, que expressa um nível de satisfação acima do ponto médio da escala Likert.

Ainda, foram acrescentadas questões que visavam obter a caracterização dos participantes, como o sexo, o setor de internação, a escolaridade, o estado civil e a hospitalização prévia.

O estudo teve enfoque no item educacional da pesquisa. $O$ domínio educacional contém sete questões, relacionadas às habilidades do enfermeiro em fornecer informações aos pacientes, responder a questionamentos, explicar cuidados e demonstrar técnicas ${ }^{10}$.

As informações foram digitadas em um banco de dados no programa SPSS versão 20. Procedeu-se a análise descritiva das variáveis quantitativas.

Esta pesquisa foi aprovada pelo Comitê de Ética em Pesquisa (CEP - UFVJM), sob o protocolo 731644/2014.

\section{RESULTADOS}

Em relação aos 223 entrevistados, 119 (53,4\%) eram do sexo masculino, 177 (79,3\%) possuíam ensino médio incompleto, 118 (52,9\%) eram casados, 114 (51,1\%) nunca tinham sido internados, conforme mostra a tabela 1.

Tabela 1. Distribuição de frequências e porcentagens, segundo as características sociodemográficas dos pacientes internados. Diamantina, MG, 2014 ( $n=223)$.

\begin{tabular}{lrr}
\hline Váriáveis & № & \% \\
\hline Sexo & 104 & 46,6 \\
Feminino & 119 & 53,4 \\
Masculino & & \\
Grau de instrução & 177 & 79,3 \\
Ensino médio incompleto & 46 & 20,6 \\
Ensino médio completo & & \\
Estado Civil & 62 & 27,8 \\
Solteiro & 118 & 52,9 \\
Casado & 26 & 11,7 \\
Viúvo & 17 & 7,6 \\
Separado & & \\
Hospitalização Prévia & 109 & 48,9 \\
Sim & 114 & 51,1 \\
Não & & \\
\hline
\end{tabular}

Conforme demonstrado na Tabela 2, na maioria das questões, a média foi superior a três, sendo a média final do instrumento 3,62 . 
Tabela 2. Distribuição das frequências e médias de satisfação dos pacientes quanto à assistência de enfermagem: dimensão educacional, Diamantina, MG, 2014 ( $n=223$ ).

\begin{tabular}{|c|c|c|c|}
\hline Instrumento de Satisfação do paciente (Educacional) & № & $\%$ & Média \\
\hline \multicolumn{4}{|l|}{ O enfermeiro não se preocupa em explicar sua doença } \\
\hline Concordo totalmente & 1 & 0,4 & \multirow{5}{*}{3,87} \\
\hline Concordo & 41 & 18,4 & \\
\hline Discordo & 158 & 70,9 & \\
\hline Discordo totalmente & 6 & 2,7 & \\
\hline Não tenho opinião & 17 & 7,6 & \\
\hline \multicolumn{4}{|l|}{ O enfermeiro explica as coisas em uma linguagem simples } \\
\hline Concordo totalmente & 1 & 0,4 & \multirow{5}{*}{3,86} \\
\hline Concordo & 19 & 8,5 & \\
\hline Discordo & 191 & 85,7 & \\
\hline Discordo totalmente & 8 & 3,6 & \\
\hline Não tenho opinião & 4 & 1,8 & \\
\hline \multicolumn{4}{|c|}{ O enfermeiro faz muitas perguntas, mas não faz nada a respeito } \\
\hline Concordo totalmente & 4 & 1,8 & \multirow{5}{*}{3,69} \\
\hline Concordo & 23 & 10,3 & \\
\hline Discordo & 173 & 77,6 & \\
\hline Discordo totalmente & 6 & 2,7 & \\
\hline Não tenho opinião & 17 & 7,6 & \\
\hline \multicolumn{4}{|c|}{ O enfermeiro deveria informar sobre os resultados dos meus exames } \\
\hline Concordo totalmente & 14 & 6,3 & \multirow{5}{*}{2,76} \\
\hline Concordo & 107 & 48,0 & \\
\hline Discordo & 82 & 36,8 & \\
\hline Dscordo totalmente & - & - & \\
\hline Não tenho opinião & 20 & 9,0 & \\
\hline \multicolumn{4}{|l|}{ É sempre fácil entender o que o enfermeiro está dizendo } \\
\hline Concordo totalmente & 25 & 11,2 & \multirow{5}{*}{3,77} \\
\hline Concordo & - & - & \\
\hline Discordo & 187 & 83,9 & \\
\hline Discordo totalmente & 5 & 2,2 & \\
\hline Não tenho opinião & 6 & 2,7 & \\
\hline \multicolumn{4}{|l|}{ O enfermeiro fornece as orientações na velocidade correta } \\
\hline Concordo totalmente & 2 & 0,9 & \multirow{5}{*}{3,87} \\
\hline Concordo & 10 & 4,5 & \\
\hline Discordo & 197 & 88,3 & \\
\hline Discordo totalmente & 6 & 2,7 & \\
\hline Não tenho opinião & 8 & 3,6 & \\
\hline \multicolumn{4}{|c|}{ O enfermeiro sempre dá explicações sobre os exames solicitados } \\
\hline Concordo totalmente & 3 & 1,3 & \multirow{5}{*}{3,50} \\
\hline Concordo & 43 & 19,3 & \\
\hline Discordo & 153 & 68,9 & \\
\hline Discordo totalmente & 4 & 1,8 & \\
\hline Não tenho opinião & 20 & 9,0 & \\
\hline Média final do instrumento & & & 3,62 \\
\hline
\end{tabular}


Dados desse domínio mostraram que 158 (70,9\%) tiveram a sua doença explicada pelos enfermeiros. Para 191 (85,7\%), os profissionais não utilizam uma linguagem simples, dificultando a compreensão; 173 (77,6\%) discordam de que o enfermeiro faça muitas perguntas sem utilidade; 187 (83,9\%) dos entrevistados não entendem o que é falado pelos enfermeiros; 197 (83,3\%) alegam não receber as informações em uma velocidade correta; $153(68,6 \%)$ gostariam que os enfermeiros dessem explicações completas e suficientes da solicitação de exames, e 107 (48,0\%), dos resultados desses.

A questão mais bem avaliada entre as positivas foi "o enfermeiro fornece as informações na velocidade correta", com média de 3,87, e, na avaliação negativa, a mais expressiva foi a média de 3,69 na questão "o enfermeiro faz muitas perguntas, mas quando ele recebe a resposta, parece não fazer nada a respeito".

\section{DISCUSSÃO}

Houve predominância de pacientes do sexo masculino, casados, com nível de escolaridade inferior a ensino médio incompleto e sem internações prévias.

Tal predominância masculina pode correlacionar-se ao fato de estes negligenciarem sua saúde tanto por falta de tempo para cuidar de si devido ao trabalho, quanto para manter a sua masculinidade, por isso procuram menos o serviço de saúde primário para prevenir doenças. Além disso, tendem a ter hábitos menos saudáveis que as mulheres ${ }^{11}$.

Outros estudos sobre a satisfação de pacientes quanto à assistência de enfermagem tiveram, em sua maioria, pessoas casadas $^{4,8,12}$ e outras com ensino médio incompleto ${ }^{4-10}$.

Nesse estudo, o nível de satisfação em relação ao item educacional alcançou uma média de 3,62. Pesquisas realizadas no ambiente hospitalar utilizando o ISP encontraram bom nível de satisfação dos pacientes no item educacional: média 4 em um hospital da Região Centro-Oeste ${ }^{8} ; 3,7$ em um hospital do interior do Estado de São Paulo ${ }^{4}$; 3,6 em um hospital de referência no cuidado à saúde da mulher, no interior do Estado de São Paulo ${ }^{12}$; 3,85 em uma Unidade de Terapia Intensiva da Unidade de Recuperação Pós - Anestésica em Porto Alegre ${ }^{10}$; 3,63 em um serviço de emergência hospitalar na região sul do Brasil $^{9}$.

Os dados mostraram que os pacientes não entendem o que o enfermeiro diz; afirmam que ele não utiliza uma linguagem simples. Os pacientes dizem ainda não receber as informações em uma velocidade correta e que gostariam de ser informados acerca dos motivos pelos quais são feitos exames e sobre seus respectivos resultados. Por outro lado, eles ressaltaram que o enfermeiro explica sobre a sua doença, são responsivos no momento de uma pergunta e atendem prontamente ao pedido de seus clientes.
A maioria dos profissionais tende a utilizar termos técnicos e científicos durante a comunicação com o paciente, dificultando a compreensão do que é dito ${ }^{13}$.

A forma com que o profissional de saúde irá estabelecer o diálogo com o paciente é crucial para que haja um bom entendimento. $\mathrm{O}$ uso de terminologias técnicas pode dar uma conotação completamente controversa ao paciente ${ }^{14}$. É necessário que o enfermeiro crie um vínculo de confiança com o seu paciente, para que haja uma abertura não só para o diálogo e escuta qualificada, mas também para a educação em saúde. Porém, tudo deve acontecer de forma clara, para que atinja o resultado esperado e preciso. Além disso, durante a conversa, é preciso que o enfermeiro faça referência ao paciente pelo nome e mantenha o contato visual ${ }^{13}$.

Mais que explicar sobre a patologia, o profissional deve-se preocupar com a velocidade com que essa informação está sendo passada. Portanto, é preciso falar pausadamente, para que haja um bom entendimento por parte do paciente, dando a ele abertura para possíveis questionamentos. É ideal que o profissional se certifique se houve o entendimento devido, pedindo para que o paciente repita as orientações recebidas. É necessário que o enfermeiro afaste de si qualquer tipo de dominação na conversa, assim o paciente terá liberdade de fazer inferências e se sentirá mais próximo do profissional e acessível a ele ${ }^{15}$.

Estudo conduzido em Recife revelou que os pacientes eram pouco informados não só sobre o seu próprio estado de saúde, mas também a respeito dos exames solicitados e realizados. Dessa forma, retira-se deles o direito de participar das decisões do próprio tratamento, ficando inteiramente à mercê da iniciativa e deliberações da equipe de saúde. Mostrou também que eles desejam conhecer seu quadro clínico e participar do tratamento ${ }^{16}$, assim como os dados apresentados por essa pesquisa.

Nota-se, portanto, que a satisfação de quem utiliza os serviços de saúde vai desde sua recepção, passando pela relação que é estabelecida com o profissional até a estrutura do local em que é atendido ${ }^{17}$.

O cuidado de qualidade exige muito do profissional, pois envolve conhecimento, habilidade e também a capacidade de se relacionar bem com o outro, de forma clara e efetiva, sendo a comunicação verbal e não verbal essencial nesse processo ${ }^{1}$.

Por fim, no sentido de repassar a responsabilidade e o autocuidado ao paciente, uma boa alternativa é a educação em saúde. Isso pode trazer benefícios ao paciente e ao profissional, influenciando diretamente na sua satisfação, pois, para que a educação em saúde aconteça, vínculos de confiança e diálogo devem ser estabelecidos. O educar e o cuidar juntos levam o paciente a repensar suas práticas de autocuidado ${ }^{6}$. Lembrando que as orientações ao paciente devem ser tanto faladas quanto 
escritas $^{18}$.

O usuário do serviço é capaz de informar a qualidade do cuidado recebido. Portanto, analisar a satisfação do paciente influencia diretamente na melhoria da qualidade da assistência, pois, a partir de seu resultado, terá a mensuração/visão do paciente sobre o serviço a ele prestado, e subsidiará tomadas de decisões tanto para as questões administrativas quanto para a própria gestão do cuidado de enfermagem ${ }^{19}$.Para a instituição, é de extrema importância que seu paciente esteja satisfeito com o cuidado que recebe. Para isso, é preciso que os seus profissionais atendam às expectativas dos pacientes. Consequentemente, cabe à instituição tanto a capacitação de seus profissionais quanto o fornecimento de um cenário propício para que a educação em saúde aconteça ${ }^{20}$.

As limitações do estudo estão relacionadas ao instrumento aplicado, ISP, que, ao ser adaptado para o Brasil, não se consideraram as três categorias profissionais da equipe de enfermagem, generalizando, nos itens do questionário, o termo "profissionais da enfermagem". Também, o ISP é um questionário fechado, dessa forma, não permite ao paciente expor anseios e sugestões.

\section{CONCLUSÃO}

Os resultados demonstraram satisfação do paciente em relação à dimensão educacional ao serviço de enfermagem.

Bons níveis de satisfação foram obtidos como em quesitos como explicações fornecidas pelo enfermeiro sobre a doença, responsividade perante solicitações e atendimento aos pacientes.

As questões de linguagem, o entendimento do que é falado e a velocidade das informações transmitidas foram os que tiveram menores índices de satisfação. Um bom método para trabalhar com essas insatisfações é a educação em saúde.

O enfermeiro é um profissional apto para promover o educar em saúde, evitando, assim, incompreensões e estimulando o cliente ao autocuidado, colocando-o como parte fundamental e imprescindível de sua própria recuperação. Uma boa comunicação também é crucial para a satisfação dos pacientes. Portanto, o cuidar, o gerenciar e o educar devem-se, continuamente, permear o cotidiano das práticas de saúde.

\section{REFERÊNCIAS}

1. Seleghim MR, Teixeira JA, Matsuda LM, Inoue KC. Avaliação de usuários sobre a qualidade dos serviços de um pronto socorro. Rev. Rene. 2010 Jul-Set; 11(3): 122-31.

2. Mendes VLS. Avaliação dos serviços de saúde pelos usuários: Questão de cidadania. Revista Baiana de Enfermagem. 2003 Jan-Ago; 18(2): 97-110. doi: http://dx.doi.org/10.18471/rbe.v18i1.3876.

3. Imbelloni LE, Pombo IAN, Morais GB Filho. Reduced fasting time improves comfort and satisfaction of elderly patients undergoing anesthesia for hip fracture. Rev. Bras. Anestesiologia. Mar-Abr 2015; 65(2): 117-123. doi: http:// dx.doi.org/10.1016/j.bjane.2013.10.019.

4. Dorigan GH, Guirardello EB. Satisfação do paciente em uma unidade de gastroenterologia. Acta paul. enferm. 2010; 23(4): 500-505. doi: http://dx.doi. org/10.1590/S0103-21002010000400009.

5. Barbosa LR, Melo MRAC. Relações entre qualidade da assistência de enfermagem: revisão integrativa da literatura. Rev. bras. enferm.2008 Maio-Jun; 61(3): 366-370. doi: http://dx.doi.org/10.1590/S0034-71672008000300015.

6. Rigon AG, Neves ET. Educação em saúde e a atuação de enfermagem no contexto de unidades de internação hospitalar: o que tem sido ou há para ser dito? Texto contexto - enferm. 2011 Out-Dez; 20(4): 812-817. doi: http:// dx.doi.org/10.1590/S0104-07072011000400022.

7. Oliveira AML. Satisfação do paciente com os cuidados de enfermagem adaptação cultural e validação do Patient Satisfaction Instrument. [dissertação]. Campinas (SP): Universidade Estadual de Campinas; 2004.

8. Freitas JS, Silva AEBC, Minamisava R, Bezerra ALQ, Sousa MRG. Qualidade dos cuidados de enfermagem e satisfação do paciente atendido em um hospital de ensino.Rev. Latino-Am. Enfermagem. 2014 Maio-Jun; 22(3): 454-60. doi: 10.1590/0104-1169.3241.2437.

9. Levandovski PF, Lima MADS, Acosta AM. Patient satisfaction with nursing care in an emergency service. Invest Educ Enferm. 2015; 33(3): 473-481. doi: 10.17533/udea.iee.v33n3a11.
10. Moraes KB. Carga de trabalho de enfermagem e satisfação dos pacientes em Unidade de Terapia Intensiva Pós-operatória [dissertação]. Porto Alegre (RS) Escola de Enfermagem da UFRGS; 2014.

11. Silva SPC, Menandro MCS. As representações sociais da saúde e de seus cuidados para homens e mulheres idosos. Saúde Soc.2014; 23(2): 626-640. doi: 10.1590/S0104-12902014000200022.

12. Odinino NG, Guirardello EB. Satisfação da puérpera com os cuidados de enfermagem recebidos em um alojamento conjunto. Texto contexto enferm. 2010 Out-Dez; 19(4): 682-690. doi: http://dx.doi.org/10.1590/S010407072010000400011

13. Schimith MD, Simon BS, Bretas ACP, Budo MLD. Relações entre profissionais de saúde e usuários durante as práticas em saúde.Trab. educ. saúde. $2011 \mathrm{Nov}$; 9(3): 479-503. doi: http://dx.doi.org/10.1590/S1981-77462011000300008.

14. Milbrath VM, Siqueira HCH, Motta MGC, Amestoy SC. Comunicação entre a equipe de saúde e a família da criança com asfixia perinatal grave. Texto contexto - enferm. 2011 Out-Dez; 20(4): 726-734. doi: http://dx.doi.org/10.1590/S010407072011000400011.

15. Dias JVS, Ferreira J. Contribuições da antropologia para o campo da educação em saúde no brasil.Trab. educ. saúde. 2015 Mar; 13(2): 283-299. doi: http://dx.doi.org/10.1590/1981-7746-sip00004.

16. Miranda ACA, Feliciano KVO, Sampaio MA. A comunicação médico-paciente na percepção de mulheres com nódulo mamário e indicação de biópsia. Rev. Bras. Saúde Mater. Infant. 2014 Jul-Set; 14(3): 251-260.doi: http://dx.doi. org/10.1590/S1519-38292014000300006.

17. Queiroz MVO, Ribeiro EMV, Pennafort VPS. Assistência ao adolescente em um serviço terciário: acesso, acolhimento e satisfação na produção do cuidado. Texto contexto - enferm. 2010; 19(2): 291-299. doi: http://dx.doi.org/10.1590/ S0104-07072010000200010.

18. Coriolano-Marinus MWL, Pavan MI, Lima LS, Bettencourt ARC.Validação de material educativo para alta hospitalar de pacientes com prescrição de 
oxigenoterapia domiciliar prolongada.Esc. Anna Nery. 2014 Abr-Jun; 18(2): 284289. doi: http://dx.doi.org/10.5935/1414-8145.20140041.

19. Al-Abri R,Al-Balushi A. Patient Satisfaction Survey as a Tool Towards Quality Improvement. Oman Med J. 2014 Jan; 29(1): 3-7. doi: 10.5001/omj.2014.02.
PubMed Central PMCID: PMC3910415.

20. Manzo BF, Brito MJM, Correa AR. Implicações do processo de Acreditação Hospitalar no cotidiano de profissionais de saúde. Rev. esc. enfermagem USP. 2012; 46(2): 388-394.

\section{Como citar este artigo/How to cite this article:}

Caldeira ABR, Baracho VS, Guedes CF, Ferreira PHC, Guedes HM, Ribeiro LCC. Satisfação de pacientes quanto à assistência de enfermagem: dimensão educacional. J Health Biol Sci. 2016 Abr-Jun; 4(4):245-250. 